\title{
L'uso del «di» e del «che» nei comparativi di diseguaglianza
}

\author{
U rsula Bedogni
}

\begin{abstract}
The great variety in the usage of the comparatives in Italian often causes problems to the Catalan- or Castilian-speaking student when faced with its practical application. In the first place, this article analyzes the structure of syntagmatic comparisons, to then move on to dealing with the temporal and modal functions required. Thus, a general chart is drawn which supplies a clarification of the contrastive situation and which, as a consequence, makes the didactic moment easier.
\end{abstract}

Un problema spesso trascurato nell'insegnamento dell'italiano come L2 è quello che riguarda l'analisi dei comparativi di diseguaglianza, la cui difficoltà - sia per gli ispanofoni che per i catalanofoni - deriva dalla coesistenza della preposizione DI e della congiunzione CHE per introdurre il secondo termine di paragone.

In generale, le varie grammatiche a disposizione non sono di grande aiuto, perché, come vedremo, propongono criteri molte volte diversi fra loro e anche piuttosto incompleti. N on esiste una regola total izzante e precisa per decidere quando usare il DI invece del $\mathrm{CHE}$ : molte grammatiche scrivono «è preferibile in tal caso... » 0 «è generalmente utilizzato nel talaltro... », 0 addirittura non forniscono nessuna spiegazione limitandosi ad addurre una serie di esempi. I criteri giustificativi più sfruttati si possono, in linea di massima, suddividere in tre gruppi.

$\mathrm{N}$ el primo rientra l'analisi dei comparativi secondo la categoria morfologica a cui appartengono entrambi i termini della comparazione.

II dizionario Zingarelli (1996), per esempio, scrive alla voce $\mathrm{CHE}$ :

Con valore comparativo introduce il secondo termine di paragone ed è in vigore quando il paragone viene fatto tra due aggettivi, o participi, o infiniti, o sostantivi e pronomi preceduti da preposizione.

Immediatamente dopo propone la frase «corre più veloce $\mathrm{CH}$ E il vento». È evidente che una spiegazione di questo tipo risulta troppo schematica e incompleta per comprendere l'effettiva complessità dei casi possibili. 
Rispetto al secondo criterio, viene considerato soltanto il secondo termine di paragone.

In D ardano-T rifone (1989: 220-221) si segnala che:

[II DI] si mette solo davanti a un nome 0 a un pronome non retti da preposizione o davanti a un avverbio:

è più giovane di Franco; correva più veloce di me; è più riflessivo di prima.

M eno propriamente si direbbe:

è più giovane che Franco; correva più veloce che me; è più riflessivo che prima.

Negli altri casi, cioè davanti a un nome o pronome retto da preposizione 0 quando si paragonano tra loro due verbi, due aggettivi o due avverbi, si può usare solo che:

lo fece più per dovere che per suo piacere;

è più facile a dirsi che a farsi;

pareva più rassegnata che persuasa;

agisce più istintivamente che razional mente.

La confusione è palese soprattutto a proposito degli avverbi, perché in una frase del tipo «O ggi sono convinto, ancor più DI ieri, di aver fatto la scelta giusta» difficilmente un italofono userebbe un $\mathrm{CH}$, eppure la comparazione avviene (almeno esplicitamente) fra due avverbi, oggi e ieri. che:

Carrera Díaz (1988: 409-410) opera un distinguo più preciso e osserva

El segundo término de la correlación presenta, como puede verse, una doble forma [... ]:

1. Se usa di cuando [... ] la marca de referencia está constituida por determinados adverbios [Più di così non ho potuto fare, 0 ggi ho lavorato più di/ che ieri, Adesso si sta meglio di/che prima [... ].

2. Se usa siempre che en cualquiera de los siguientes casos: [... 2.5. Dos adverbios en -mente: Lavora più intelligentemente che costantemente.

E anche in Serianni (1991: 210-211) troviamo, anche se in maniera meno articolata, che:

La preposizione di si adopera di preferenza quando:

[...]

b) il secondo termine è un avverbio: «più esperto di prima»; «più stupidi di così si muore» $[\ldots]$ 
Si adopera invece la congiunzione che quando:

[...]

c) si paragonano fra loro parti del discorso che siano aggettivi (avverbi, verbi): «'ha detto più per scherzare che per offenderti», «mi piace meno ora che prima» (ma si può dire anche «meno ora di prima» [... ]).

Per Renzi-Salvi (1988) questo secondo criterio è particolarmente dominante: ogni volta che viene analizzata la comparazione sui vari sintagmi, si inizia sempre la spiegazione sostenendo che «l secondo termine di paragone è un Sintagma $X$ introdotto da... ». M a lascia un po' perplessi il fatto che, analizzando la comparazione su sintagma nominale, venga proposto come esempio «gli ospiti hanno mangiato più dolci CHE salatini» adducendo la seguente giustificazione:

II secondo termine può essere un SN virtualmente inserito in una frase identica alla principale, non espressa, in cui occupa la stessa posizione ed ha la stessa funzione grammaticale; il secondo termine di paragone è allora introdotto da che. ${ }^{1}$

A questo proposito sembra più chiara la spiegazione fornita da Carrera D íaz che, nell'elenco dei casi in cui è obbligatorio l'uso della congiunzione $\mathrm{CHE}$, inserisce anche:

D os sustantivos con respecto a la cantidad: H o più libri che dischi. ${ }^{2}$

Per questo secondo gruppo, comunque, è possibile stabilire una serie di regole comuni per decidere quando usare $\mathrm{CHE}$ davanti al secondo termine di paragone:

- davanti agli aggettivi (tranne i numerali);

- davanti ad un'azione verbale;

- davanti ad un gruppo preposizionale;

- davanti ai nomi che rappresentano quantità.

La preposizione D I si potrebbe utilizzare, ma mai in maniera obbligatoria, davanti ai nomi e ai pronomi non preceduti da una preposizione (sempre che i nomi non esprimano una quantità, come nella frase «in questo bicchiere c'è più acqua $\mathrm{CHE}$ vino»).

Tuttavia, nemmeno questo secondo criterio è soddisfacente, perché, anche nei casi in cui è consigliato DI, spesso è presente $\mathrm{CH} E$, come nella frase «è più pieno il mio bicchiere CHE il tuo».

2. Carrera Díaz (1988: 410). 


\section{III}

L'analisi può avvenire anche secondo un terzo criterio, cioè basandosi sull'oggetto della comparazione che può avvenire tra:

1. due termini rispetto ad una stessa qual ità (es. «M ario è più sveglio D I te»);

2. due qualità rispetto allo stesso termine (es. «G iuseppe è più stupido $\mathrm{CH} \mathrm{E}$ cattivo»).

Q uesto criterio è utilizzato da Fogarasi (1984: 207), dove leggiamo:

Paragonando due persone 0 cose, come termine di paragone è preferibile di, ma che può anche essere impiegato: II tuo racconto è più interessante di questo romanzo. M a va bene anche... più interessante che questo romanzo.

$Q$ uando invece si paragonano due qualità, il termine di paragone è sempre che: Q uella ragazza è più buona che bella.

Che si ottiene in via di ellissi da che non sia (di uso raro ormai), cioè di una proposizione subordinata comparativa.

Lo stesso discorso vale per Battaglia-Pernicone (1960: 167-168) e, in parte, è ripreso anche da Serianni (1991: 211):

Si adopera la congiunzione che quando [tra le altre cose] si mettono a raffronto non due nomi caratterizzati dall'aggettivo qualificativo ( $\ll M$ ario è meno religioso di Gino») ma due qualità riferite in misura differente allo stesso nome (che funge, da termine di paragone rispetto a se stesso): «M ario è più furbo che intelligentes.

Carrera D íaz (1988: 409-410) è più preciso e specifica quanto segue:

1. Se usa di cuando se compara una cualidad, circunstancia o modo de acción con respecto a dos portadores de la misma: Luigi è più alto di Pietro.

2. Se usa siempre che en cualquiera de los siguientes casos: [... ] 2.1. C uando se comparan dos cualidades con respecto a un mismo portador de ambas: Luigi è più al to che intelligente.

II problema si presenta sempre nel primo caso, quando non è detto che non si possa usare il $\mathrm{CHE}$.

$\mathrm{M}$ a per risolvere la questione deve essere impostata correttamente la domanda: è funzionale, nell'insegnamento della lingua agli stranieri, adottare questi criteri come ci vengono presentati, in maniera se non confusa sicuramente dispersiva, o è possibile elaborare un criterio più generale, più facilmente individuabile anche dagli studenti, senza costringerli ogni volta ad analizzare grammaticalmente le frasi?

A nostro giudizio, un criterio metodologico funzionale è quello adottato da Brunet (1984: 123-202). Invece di utilizzare espressioni come «ter- 
mine» e «qualità», viene definito comparante l'elemento attivo che determina la comparazione e comparato l'elemento passivo oggetto della comparazione, anche qualora si tratti d'un soggetto o di un complemento d'oggetto diretto.

Possono quindi esserci frasi con un solo comparante e due comparati, che rientrerebbero in un cosiddetto Schema $\mathbf{A}$, e frasi con due comparanti e un solo comparato che rientrerebbero nello Schema B.

Ad esempio, nella frase «Pietro è più furbo DI Paolo», I'unico comparante è la furbizia (Schema $\mathbf{A}$ ), mentre nella frase «il mare era più verde $\mathrm{CHE}$ azzurro» |'unico comparato èil mare (Schema B).

Allo schema $B$ appartengono anche quelle frasi in cui i sostantivi esprimono una quantità: «M ario ha più soldi $\mathrm{CHE}$ soddisfazioni», dove $\mathrm{M}$ ario è visto prima in relazione ai soldi e poi alla soddisfazione.

Lo stesso discorso vale anche per «ho più sete $\mathrm{CHE}$ fame», che potrebbe esser perfettamente trasformata in «sono più assetata $\mathrm{CH}$ E affamata».

N ella frase «è più facile dirlo CHE farlo», con i verbi all'infinito, I'unico comparato sarebbe un personaggio indeterminato, dal momento che la frase è impersonale, considerato prima in rapporto al dire e poi al fare.

Infine, la proposizione «'uomo ama più tradire gli amici $\mathrm{CHE}$ i nemici», presenta un comparato unico, I'uomo, e un secondo termine retto da un CH E nonostante si tratti di un sostantivo preceduto addirittura da un determinante.

Viceversa, nella frase «Anna è più giovane $D$ I Paola», il soggetto del verbo non è più l'unico comparato: i comparati sono due, Anna e Paola, in rapporto all'unico comparante che è espresso dall'aggettivo giovane. Q uindi, in questo caso è il D I a sostituire un'eventuale proposizione ellittica.

Riassumendo, CHE è sempre usato nello schema $B$, mentre lo è raramente nello schema $A$, dove invece prevale l'uso del $D I$.

Anche nel caso dei pronomi personali, malgrado la Grande Grammatica di Consultazione ripeta in continuazione che davanti ai pronomi personali non preceduti da preposizione non può essere usato $\mathrm{CHE}$, possiamo trovare un comparato unico in relazione a due comparanti anche con un pronome personale complemento d'oggetto diretto: «ottenere quel lavoro avrebbe aiutato più te $\mathrm{CHE}$ lui».

Un esempio più chiaro è dato dalle frasi:

1. «Paola ama lui più CHE te»;

2. «Paola ama lui più DI te».

N ella prima l'unico comparato è Paola vista in relazione ai due comparanti lui e te, mentre nella seconda i comparati sono due Paola e te e quindi in questo caso si userebbe la preposizione D I.

Un ultimo esempio è il proverbio «meglio un asino vivo $\mathrm{CHE}$ un dottore morto», dove i comparati non sono due, l'asino e il dottore, ma uno soltanto, cioè la persona che si trova posta di fronte all'alternativa. 
In conclusione, crediamo che quest'ultimo metodo proposto sia l'unico veramente completo e adatto a risolvere la «questione comparativi» e a dotare gli studenti di uno strumento comprensibile senza per questo impoverire la lingua italiana di una propria peculiarità. ${ }^{3}$

\section{Bibliografia}

Battaglia, S.; Pernicone, V. (1960). La grammatica italiana. Torino: Loescher.

BRUNET, J. (1984). Grammaire critique del'italien. U niversité de Paris VIII.

Carrera DíAz, M . (1988). Curso delengua italiana. Vol. I. Barcellona: Ariel.

D ARd AN O, M.; T RIFONE, P. (1989). Grammatica italiana. Bologna: Zanichelli.

FERRARI, A. (1996). «Vent'anni di studi sulla subordinazione avverbiale. Un pano-

rama bibliografico». Studi italiani di linguistica teorica e applicata, 1: 220-223.

FogarasI, M . (1984). Grammatica italiana del '900. Roma: Bulzoni.

RenzI, L.; SALVI, G. (1988). Grande Grammatica italiana di consultazione Vol. II. Bologna: Il Mulino.

SERIAN NI, L. (1991). Grammatica italiana. Torino: UTET.

3. Per un ulteriore aggiornamento bibliografico, cfr. Ferrari (1996: 220-223). 